\title{
Diagnosis and phylogenetic analysis of ovine pulmonary adenocarcinoma in China
}

\author{
Keshan Zhang $\cdot$ Hanjin Kong $\cdot$ Yongjie Liu • \\ Youjun Shang $\cdot$ Bin Wu $\cdot$ Xiangtao Liu
}

Received: 10 July 2013/ Accepted: 24 September 2013/Published online: 23 October 2013

(C) The Author(s) 2013. This article is published with open access at Springerlink.com

\begin{abstract}
Ovine pulmonary adenocarcinoma (OPA) is a lung tumor of sheep caused by jaagsiekte sheep retrovirus (JSRV). OPA is common in sheep, and it is most commonly observed in China. Without preventative vaccines and serological diagnostic tools for assay of OPA, identification of JSRV based on reverse transcription polymerase chain reaction (RT-PCR) is very important for prevention and control measures for OPA in practice management. In this study, the diagnosis of OPA was made from analysis of clinical signs, pathological observations, JSRV-like particle discovery, and RT-PCR of the target env gene. The phylogenetic analysis showed that the China Shandong (SD) strain studied in this article belonged to exogenous JSRV, and it was very similar to $92 \mathrm{k} 3$, which was isolated from sheep in the Kenya (Y18305). The current study reported a severe outbreak of OPA in Shandong Province, China. The observations could offer a comparative view of the env gene of JSRV.
\end{abstract}

Keshan Zhang and Hanjin Kong contributed equally to this work.

K. Zhang $\cdot$ Y. Liu $\cdot$ Y. Shang $\cdot$ X. Liu $(\bowtie)$

State Key Laboratory of Veterinary Etiological Biology,

National Foot-and-Mouth Disease Reference Laboratory, Lanzhou Veterinary Research Institute, Chinese Academy of Agricultural Sciences, Xujiaping No.1, Yanchangpu, Lanzhou 730046, Gansu, People's Republic of China

e-mail: hnxiangtao@hotmail.com

K. Zhang

e-mail: zks009@126.com

H. Kong · B. Wu

State Key Laboratory of Agricultural Microbiology, College of Veterinary Medicine, Huazhong Agricultural

University, Wuhan 430070, Hubei, People's Republic of China
Keywords China $\cdot$ Molecular identification · Ovine pulmonary adenocarcinoma $\cdot$ Phylogenetic analysis

\section{Introduction}

Ovine pulmonary adenocarcinoma (OPA), also known as ovine pulmonary adenomatosis and jaagsiekte, is a contagious neoplasm affecting the lungs of sheep, which is caused by jaagsiekte sheep retrovirus (JSRV) [1-3]. JSRV has been described in various breeds of sheep, rarely in goats, and does not appear to infect other animals $[4,5]$. OPA is believed to be the most important disease that can affect international trade as determined by the OIE (OIE Manual, 7th Edition). The disease is characterized by fluid accumulation in the lungs, which results from the production of excess secretions by tumor cells. Filtered lung fluid from infected sheep contains viral particles, and this fluid is able to manifest the disease when inoculated intratracheally into healthy sheep [6]. OPA is the most common pulmonary tumor that affects sheep and occurs in many countries around the world, with the exception of Australia, New Zealand, and Iceland. In addition, studies on OPA are an excellent model to translate such findings to human lung carcinogenesis [7].

JSRV is classified as a beta-retrovirus and resembles a simple retrovirus, since its genomic organization contains only the essential genes characteristic of retroviruses: gag, pro, pol, and env [8]. Enzootic nasal tumor virus (ENTV) is the causative agent of contagious ovine and caprine nasal adenocarcinoma (ONA and CNA), which has the similar genome structure and shares high homology with JSRV. As we know, there are two ONA cases in Inner Mongolia, northern China [9], and southwestern China, 
respectively [10]. The JSRV env gene encodes the $\mathrm{N}$-terminal surface (SU) and C-terminal transmembrane (TM) domains of the envelope protein (Env), which is linked by disulfide bonds and embedded in the membrane. After Env cleavage by cellular furin protease, the Env protein binds the cell receptor during infection [11]. The molecular mechanism responsible for transformation has been investigated extensively and involves the YXXM motif and other Env protein domains as we have elaborated below.

There are 15-20 copies of endogenous jaagsiekte sheep retrovirus (enJSRV) are present in the genome of sheep and goats $[12,13]$. All the enJSRVs have similar gene structure with exogenous JSRV (exJSRV) and ENTV. It has been reported that there are five enJSRVs that have $85-89 \%$ sequence identity to exogenous JSRV. Regions of sequence divergence reside in the U3 region of the LTR and three regions in Gag and Env, termed variable regions, 1, 2, and 3 (VR1-3) [14]. None of the EnJSRV envs contains the YXXM motif in the $\mathrm{CT}$ region of the Env TM domain and is unable to induce transformation [15]. Though enJSRV env sequences are not oncogenic, it should be noted that the expressions of enJSRV proteins have been shown to block infection by exogenous JSRV [16].

There are currently no vaccines available for this virus, and consequently, disease control relies on regular flock inspections and prompt culling of suspected cases and, in the case of ewes, their offspring. During the long incubation period, the animals remain clinically healthy so that early stages of the disease with evidence of minor respiratory involvement can be easily confused with other respiratory diseases, and even by the most experienced clinicians [17]. Thus, developing a rapid and specific diagnostic assay is an urgent priority and necessary for control or even eradication of OPA. There is also no known specific antibody response following JSRV infection [18, 19]. Consequently, there is no diagnostic serological test currently available. At present, diagnosis depends on clinical and pathological investigations of OPA in combination with identification of the infectious agent by PCR targeting the JSRV specific gene [17].

Many sheep are bred in China's Shandong Province, which are the major sheep producing region of China. In the present study, an outbreak of OPA was diagnosed based on the clinical signs of disease, pathological investigation, and RT-PCR targeted analysis of the JSRV specific gene. The full-length env gene of JSRV, which was identified from sheep in the Shangdong Province area of China, was cloned and DNA sequenced. This represents the phylogenetic analysis of JSRV that was located in China compared with other isolated strains from around the world.

\section{Materials and methods}

Biopsies sample treatment

Biopsies of the lung were fixed in $10 \%$ buffered formalin for hematoxylin and eosin $(\mathrm{H} \& \mathrm{E})$ staining and examination by light microscopy. For transmission electron microscopic (TEM) observation, biopsies were soaked in $5 \%$ glutaraldehyde. RNA was extracted from the lungs and froth and stored at $-70{ }^{\circ} \mathrm{C}$ until used for RT-PCR.

\section{H\&E staining and TEM observation}

Sections at $4 \mu \mathrm{m}$ in thickness were obtained using a histotome, following which the tissue samples were trimmed, dehydrated, embedded in paraffin, stained with H\&E, and assessed by light microscopy [20]. Histopathological changes were analyzed by optical microscopy (OM; CX41, Japan) as described previously [21, 22]. Organelle-level changes in lung lesions were determined by transmission electron microscopy (TEM; JEM-2100, Japan), and sections were prepared and observed according to the previously published methods $[23,24]$.

\section{RNA extraction and RT-PCR}

Total RNA from lung tissues and froth was extracted using an RNeasy Kit (QIAGEN) according to the manufacturer's instructions and used as a template (the RNA treated with DNase before reverse transcription) in the RT-PCR assays. Specific primers for the exogenous OPA env gene were designed by the premier- 5 software program and synthesized in the Shanghai Sangon Biological Engineering Technology and Services Co., Ltd., China. The forward primer sequence was TTCAGCAGCCCAGCGATTT. The reverse primer sequence was AGGGAGCTTAGGTACT TGTCC. RT-PCR for the env gene was performed according to conventional methods. The RT-PCR products of env were visualized by ethidium bromide staining of $1 \%$ agarose gels that were resolved by electrophoresis and viewed under ultraviolet light.

Sequencing alignment and phylogenetic analysis

The RT-PCR products of the env gene were then cloned into the pMD-18T expression vector (Takara, Dalian, China) and transformed into E. coli DH5a. Sequencing was conducted using an automated DNA sequencer (model 3770; Applied Biosystems, USA). Complete sequences of the env gene were submitted to the NCBI GenBank database (named China SD SPA) and assigned accession numbers (KC691273). Sequence editing was performed by using the DNAstar program (http://www.dnastar.com) [25, 26]. The 
env gene sequences were collected from GenBank (http:// www.ncbi.nlm.nih.gov). Multiple alignments were produced using the ClustalW program (http://www.clustal.org) [27]. A phylogenetic tree was then constructed based on the env gene sequences (Table 1) using the neighbor-joining procedure [28, 29] using the MEGA version 4.0 software program (http://www.megasoftware.net) [30].

\section{Results}

Clinical signs and necropsy

Jaagsiekte outbroke in a farm containing 400 Dorper sheep for the purposes of breeding production, in the Shandong Province of China in 2010. All the sheep were not imported from other place, and they were bred there over many generations. Clinical symptoms of OPA were observed and characterized by a fever persisting for a period of time, with even foaming developing at the mouth. Respiratory signs occurred during the acute stages of the disease, which included malodorous, mucopurulent nasal discharge, frequent sneezing, increased respiratory rate, extended head, and mouth breathing. The sheep that presented with clinical symptoms of OPA were mostly more than 2-years old.
Serious lung swelling, necrotizing fester stove, grayish nodules, or extensive solid tumors were also found at necropsy of the lungs. The trachea and bronchus were filled with white froth. Morbidity of this case was estimated as $3.5 \%$ (14/400 cases), and mortality rates were estimated as approaching $100 \%$. In fact, 14 sheep out of 400 were showed the OPA typical clinical symptoms. Only the representative one was selected and necropsied. White froth and lung samples from the infected sheep were collected according to the Declaration of Helsinki and the Guide for the Care and Use of Laboratory Animals (Ministry of Science and Technology of China, 2006).

Mucopurulent white froth nasal discharges of infected sheep were observed when the hind legs were lifted or drifted long time. (Fig. 1a), and were found in the bronchi at necropsy (Fig. 1b). Serious lung swellings, gray-colored nodules, and extensive solid tumors were found at necropsy in the lungs (Fig. 1c).

Pathologic and electron microscopic findings of OPA

After H\&E staining of the lungs, which were obtained from infected sheep, proliferation of alveolar epithelial cells was confirmed, which were found to burst into the alveolar cavity like the papillary. In addition, lymphocytic infiltration into

Table 1 Detailed information of $e n v$ genes used in this study

\begin{tabular}{|c|c|c|c|c|}
\hline S. no. & Exogenous JSRVs & Country & Accession number & Host species \\
\hline 1 & JSRV21 & UK & AF105220 & Sheep \\
\hline 2 & JS7 & UK & AF357971 & Sheep \\
\hline 3 & China-SD SPA & China & KC691273 & Sheep \\
\hline 4 & NM & China & JQ837489 & Sheep \\
\hline 5 & JSRV-SA & South Africa & M80216 & Sheep \\
\hline 6 & $809 \mathrm{~T}$ & UK & Y18302 & Sheep \\
\hline 7 & $83 \mathrm{RS} 28$ & USA & Y18303 & Sheep \\
\hline 8 & $84 \mathrm{RS} 28$ & USA & Y18304 & Sheep \\
\hline 9 & $92 \mathrm{k} 3$ & Kenya & Y18305 & Sheep \\
\hline S. no. & Endogenous JSRVs & Country & Accession number & Host species \\
\hline 10 & enJS5F16 & South Africa & AF136224 & Sheep \\
\hline 11 & enJS56A1 & South Africa & AF153615 & Sheep \\
\hline 12 & enJSRV-NM & China & DQ838493 & Sheep \\
\hline 13 & enJSRV-16 & USA & EF680300 & Sheep \\
\hline 14 & enJSRV-9 & USA & EF680316 & Sheep \\
\hline 15 & enJSRV-4 & USA & EF680317 & Sheep \\
\hline S. no. & ENTVs & Country & Accession number & Host species \\
\hline 16 & ENTV-2 & Spain & AY197548 & Goat \\
\hline 17 & Sheep TNO28 & Spain & Y16627 & Sheep \\
\hline
\end{tabular}

Alignment of the envelope amino acid sequences of 12 exogenous JSRVs. JSRV21, JS7, China-SD SPA, NM, JSRV-SA, 809T, 83RS28, $84 \mathrm{RS} 28,92 \mathrm{k} 3$ that maintain an open reading frame along the entirety of env 

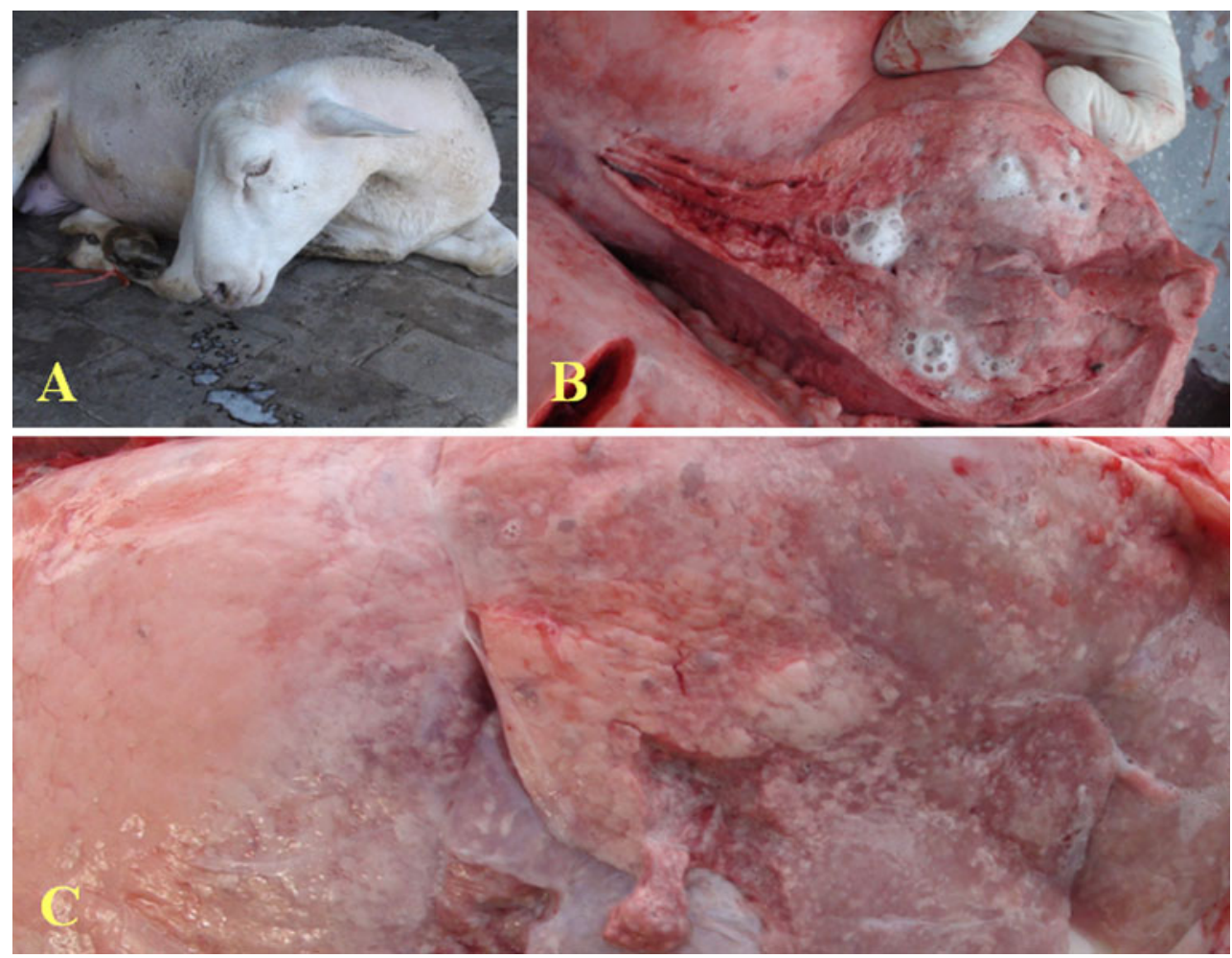

Fig. 1 Clinical and necropsy characteristics of OPA. a OPA-affected animals developed progressive respiratory distress, reflected by a white mucopurulent froth nasal discharge. b Froth was found in the

the pulmonary interstitia was observed (Fig. 2). Abundant proliferative collagen fibers were also observed in the alveolar mediastinum (Fig. 3a). OPA virus-like particles were found in the lung, and the length of the virus diameter was

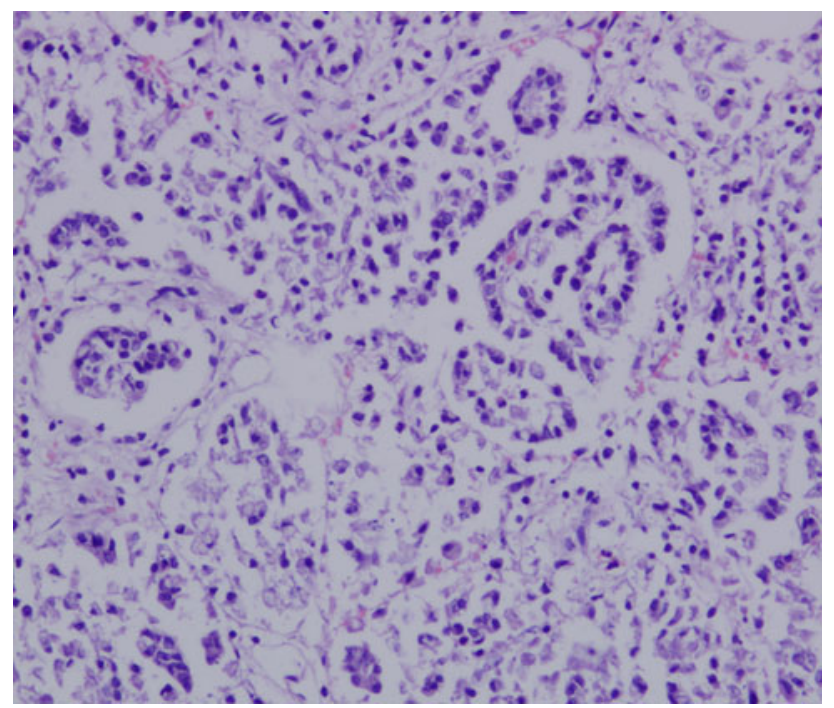

Fig. 2 Histological observations after H\&E staining of lung from infected sheep. Alveolar epithelial cell proliferation and lymphocyte infiltration in pulmonary interstitial cells were observed (H\&E staining procedure, $\times 200$ magnification) bronchus at necropsy. $\mathbf{c}$ The lungs displayed lesions characteristic of OPA, and swelling, grayish nodules, necrotizing fester stove, and extensive solid tumors (Color figure online)

determined to be approximately $100-125 \mathrm{~nm}$, with small bump-like structures existing on the surface of the virus particles by TEM analysis (Fig. 3b).

Gene amplification of env and sequencing

The expected 1,848 bp PCR fragment was visualized from total RNA extracted from infected sheep lungs, whereas no bands were observed in the negative controls. The sequencing results showed that the env gene had a size of $1,848 \mathrm{bp}$ and consisted of 615 encoded amino acids with an average $\mathrm{G}+\mathrm{C}$ ratio of approximately $40.37 \%$ and a predicated molecular weight of $69.4 \mathrm{kDa}$. The "YXXM" motif was found in the env gene (aa 462-505) and located in the region of the transmembrane area (TM). The env gene sequences identified in this study were submitted to the NCBI GenBank and assigned appropriate accession numbers (KC691273) and named China SD SPA.

Phylogenetic analysis

The results of sequencing alignment showed that the homology of the China SD SPA shared 93.7-96.6\% sequence identity at the nucleotide level and 95.8-99.5\% homology at the amino acid level compared with other 


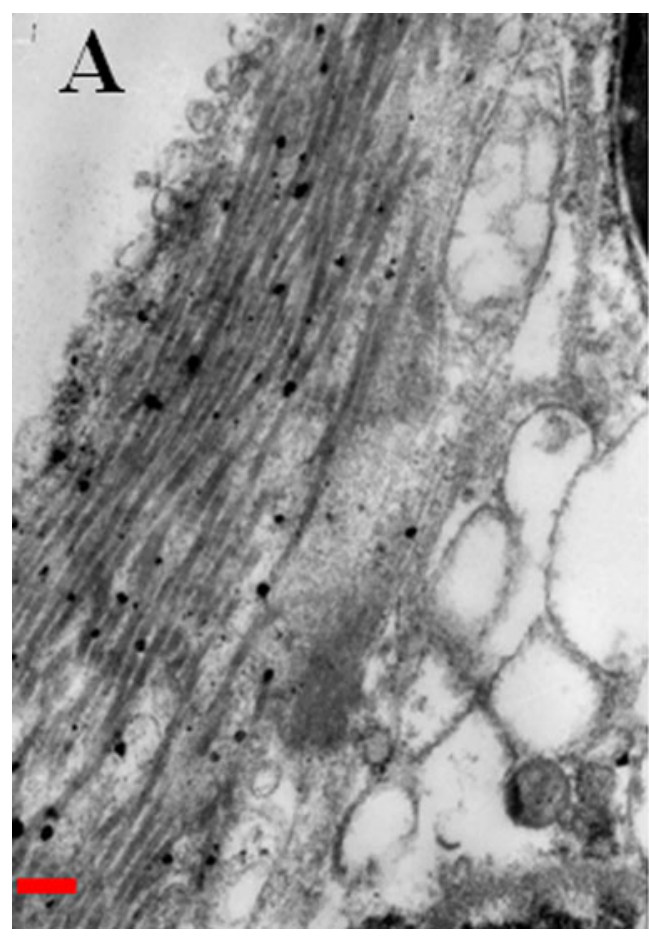

Fig. 3 Electron microscopic observation of the lung from infected sheep. a Proliferation seen in collagen fibers of the alveolar mediastinum $(\times 20,000$ magnification $)$. b OPA virus-like particles were found (arrowed), the virus diameter was found to be

exJSRV isolates from different regions of China. The nucleotide sequences of env obtained from China SD SPA shared a $96.6 \%$ similarity with a representative strain from South Africa (Accession No. NC-001494) and a 95.0\% similarity with a representative strain from UK, JSRV21 (Accession No. AF105220). Sequence analyses of the nucleotides showed that the China SD SPA had a lower homology with endogenous env. For example, the China SD SPA shared only an 88.1-89.1\% similarity with endogenous env at the nucleotide level and a 90.7-92.5\% homology at the amino acid level. The China SD SPA strain shared the lowest homology with enJSRV, which was identified in China (DQ838494) at the deduced amino acid level. The China SD SPA strain shared a respective 89.9-89.1 \% homology with Enzootic nasal tumor virus strains ENTV-2 (AY197548) obtained from goat, and TNO28 (Y16627) obtained from sheep at the amino acid level.

The phylogenetic tree data showed that the env genes of both exJSRV and enJSRV, which were both isolated from different origins, were divided into two different branches (Fig. 4). It showed that among exJSRV strains, the China SD SPA strain was the closest in similarity to $92 \mathrm{k} 3$, which was isolated from sheep in the Kenya (Y18305) (Fig. 4).

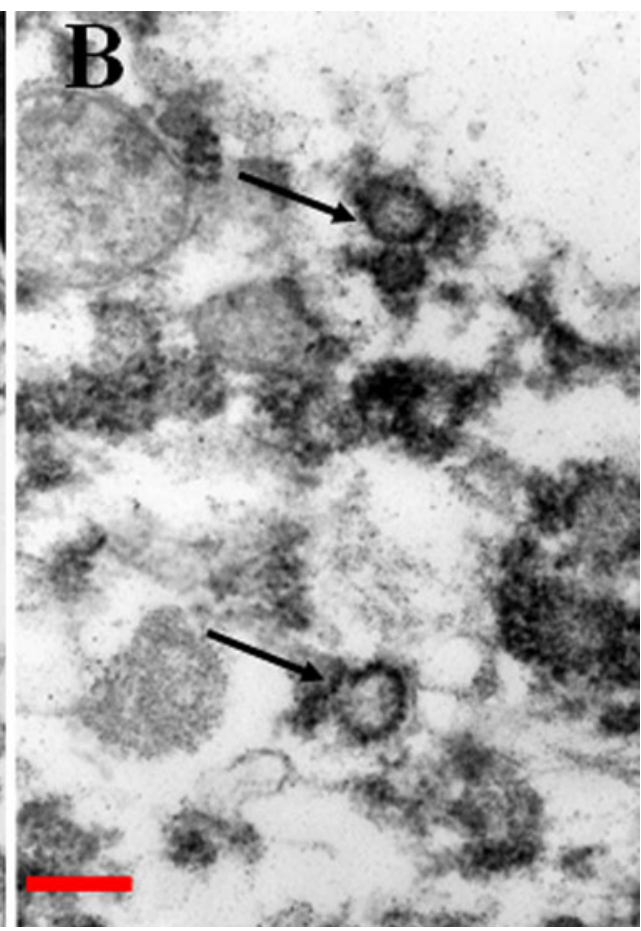

approximately $100-125 \mathrm{~nm}$, and raised small bump-like structures were found on the surface of virus particle. The red scale bar represents $200 \mathrm{~nm}$ (Color figure online)

\section{Discussion}

The methods of intensive sheep breeding are an inevitable trend, yet it provides a convenient mechanism for the spread of disease [31, 32]. OPA was first found in South Africa in 1825 [33], and it was first reported in China in 1951 [34]. Since then, OPA has been found in a variety of breeds in many countries. Not all sheep that are infected with JSRV can develop OPA [35, 36], and some breeds may be more resistant to OPA than others. OPA is an intriguing disease that presents some difficult challenges for control measures, and accounts for almost $70 \%$ of all sheep tumors [5]. The more concerning feature of JSRV infection is that it provides conditions that favor secondary infection. OPA was previously confirmed to vertically infect from mothers to their young via the colostrum [37]. Thus, OPA is an important economic and animal welfare concern requiring comprehensive prevention and control of the disease, which is an urgent need. Shandong Province is the main sheep-breeding region of China located in the eastern coastal areas. In this study, a natural outbreak of OPA among sheep was found, and JSRV was analyzed in Shandong Province.

The typical clinical signs and macroscopic lesions of OPA were observed in infected sheep in the present study 
Fig. 4 Phylogenetic analysis of different JSRV strains based on the env gene. The phylogenetic relationship was constructed using the neighbor-joining program of MEGA 4.0. Bootstrap analysis was performed with 1,000 trials. The red spot represents that the China SD SPA was the JSRV strain used in this study (Color figure online)

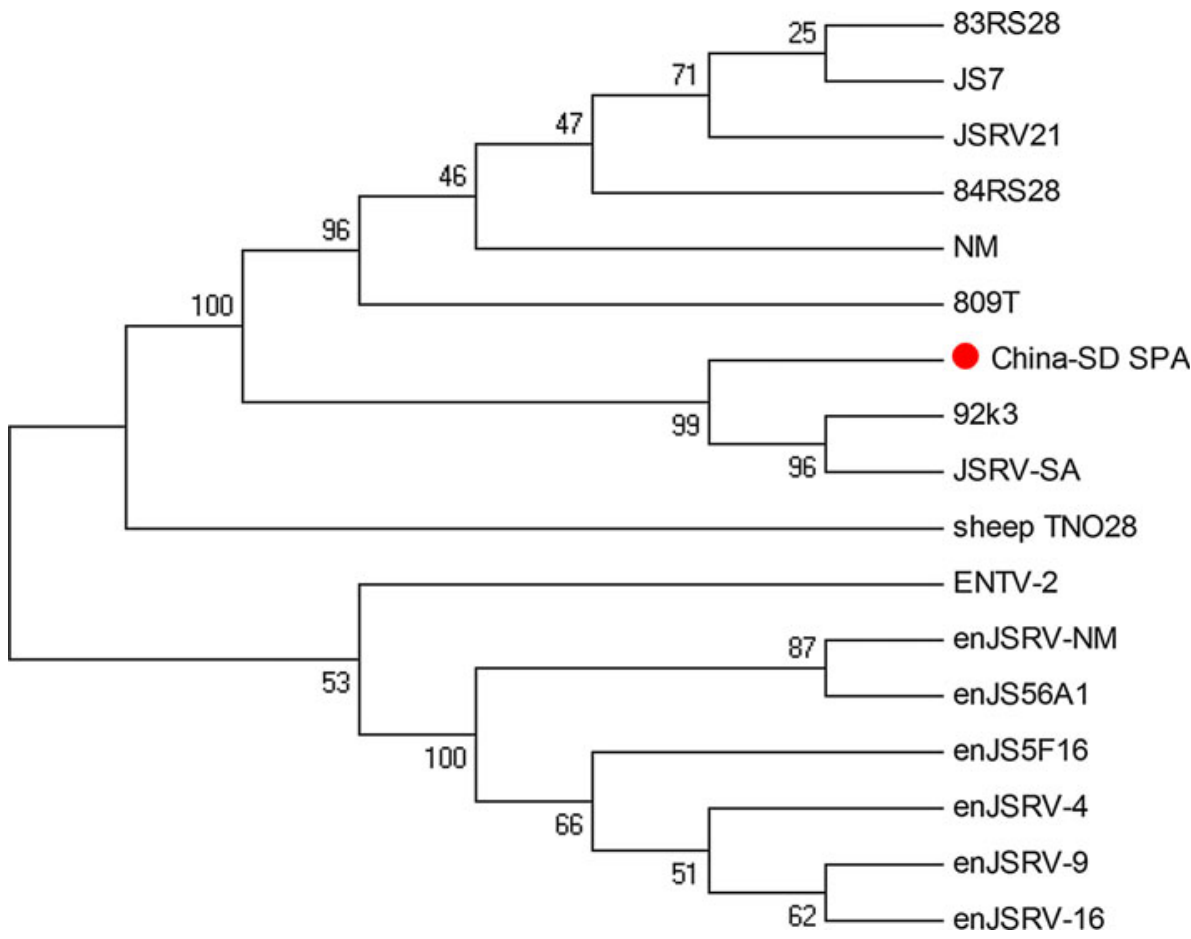

(Fig. 1a-c), which were consistent with previous reports [4, 8, 32]. Tumor characteristics were found in the lungs from infected sheep by $\mathrm{H} \& \mathrm{E}$ staining and observation (Figs. 2, 3a). Mycoplasma ovipneumoniae [38], Maedivisna [39], and other pathogens can manifest similar clinical signs as those presented by OPA. For the above reasons, OPA diagnosis was based only on clinical symptoms and was thus considered to be rather inaccurate. Up to now, JSRV can replicate for a short period of time in tumor cells from young lambs [40], and ovine choroid plexus (CP) cells can be used for a permissive cell culture system for JSRV [41]. Antibody responses to JSRV have not been detected in the sera of affected sheep, even when using highly sensitive assays such as immunoblotting or enzymelinked immunosorbent assays [19, 42]. Thus, methods of virus isolation and serological testing are not suitable for the diagnosis of OPA. To verify that JSRV existed in the lungs of infected sheep, JSRV-like particles were observed under TEM (Fig. 3b). The env gene was confirmed in the lungs of infected sheep by RT-PCR analysis.

To analyze the molecular features of JSRV, the env gene was sequenced, which showed that the env gene that was cloned in this study belonged to exogenous JSRV. Sequence results comparison between exJSRV and enJSRV env proteins indicated that TM is conserved except variable region 3 (VR3) that consists of membrane-spanning region and CT [15]. The TM area was often used in genetic evolution analyses between different strains. The domains of JSRV Env involved in the transmembrane have been studied extensively [43-45]. There is a tyrosine residue in the JSRV CT at position 590, while the CT of enJSRV lacks tyrosines.Y590 in the CT is important for JSRV Env transformation. The amino acid sequence surrounding Y590 is YRNM, and if Y590 is phosphorylated it could potentially bind cellular proteins with $\mathrm{SH} 2$ domains. YXXM and YXN are putative binding motifs for the SH2 domains of the PI3K regulatory subunit (p85) and growth factor receptor binding protein-2 (Grb-2), respectively [46]. It might be the YXXM in the JSRV CT bind to PI3K leading to downstream signaling to result in cellular transformation. Mutations of the YXXM motif can abolish or decreased transformation in cellular assays [47, 48]. One may thus conclude that the YXXM motif can be an identification criteria used to distinguish exJSRV and enJSRV. The YXXM motif that was found in the env gene identified from this case can help us conclude that the China SD SPA belonged to the exJSRV.

Sequences of the env gene obtained from 21 JSRV strains were collected from GenBank (Table 1). Sequence analysis based on both amino acid and nucleotide levels indicated that the China SD SPA strain studied in this article was very close to 92k3 (Y18305). Phylogenetic analysis showed that endogenous and exogenous JSRVs belonged to different branches, respectively (Fig. 4). The exJSRV, ENTV, and enJSRV are closely related beta retroviruses with high sequence similarity. The Envelope amino acid sequences of nine exogenous JSRVs are shown (Fig. 5), and cytoplasmic tail (CT) region and YXXM motif should be highlighted, which will help us compare exact sequences among all exogenous strains derived from 


\begin{tabular}{|c|c|c|}
\hline JSRV21 & 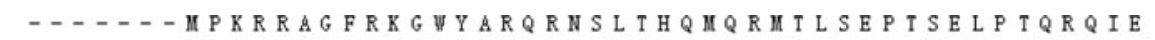 & 43 \\
\hline JS? & 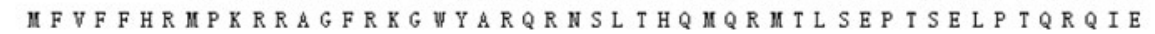 & 50 \\
\hline China-SD SPA & 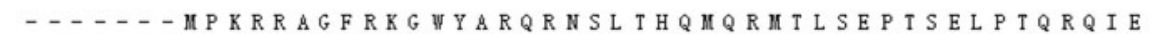 & 43 \\
\hline $\mathrm{NM}$ & 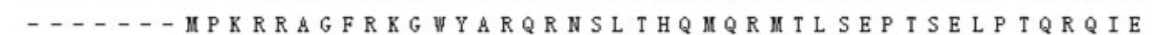 & 43 \\
\hline JSRV-SA & $-----\mathbb{M}$ PKRRAGFR $\mathbb{R}$ ARQRNSLTHQMQRM TLSEP TSELPTQRQIE & 43 \\
\hline $809 \mathrm{~T}$ & MF V F & 50 \\
\hline 83RS28 & M F VFFH MPKRRAGFR & 50 \\
\hline $84 \mathrm{RS} 28$ & 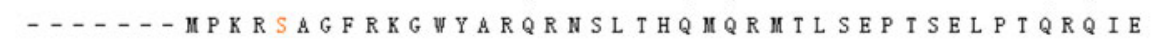 & 43 \\
\hline $92 \mathrm{k} 3$ & 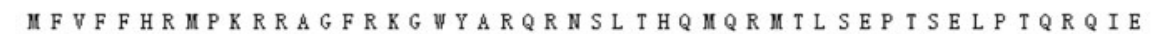 & 50 \\
\hline JSRV21 & 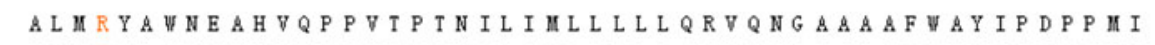 & 93 \\
\hline JS? & 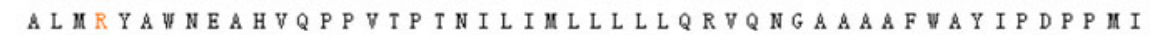 & 100 \\
\hline China-SD SPA & 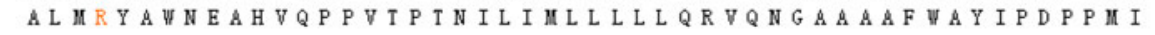 & 93 \\
\hline NM & 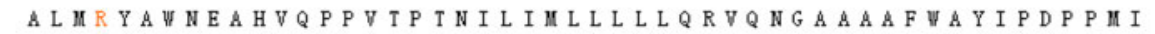 & 93 \\
\hline JSRV-SA & 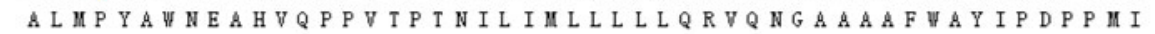 & 93 \\
\hline $809 \mathrm{~T}$ & 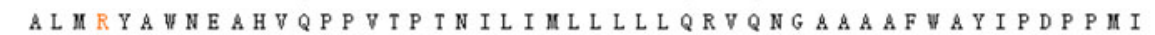 & 100 \\
\hline 83RS28 & 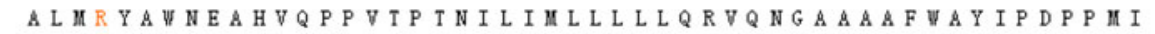 & 100 \\
\hline $84 \mathrm{RS} 28$ & 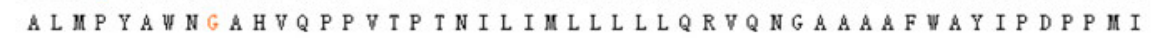 & 93 \\
\hline $92 \mathrm{k} 3$ & 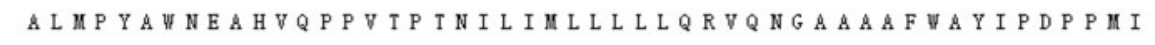 & 100 \\
\hline JSRV21 & QSLG WDREIVPVYVND TSLLGGKSDIHISPQQAN ISFYGL T TQYP MCF SY & 143 \\
\hline JS? & QSLG WDR I & 150 \\
\hline China-SD SPA & QSLGWDREIVPVYVNDISLLGGKSDIHISPQQANISFYGLTTQYPMCFSY & 143 \\
\hline $\mathrm{NM}$ & QSLGWDREIVPVYVNDTSLLGGKSDIHISPQQANISFYGLITQYP M CFSY & 143 \\
\hline JSRV-SA & QSLGWDREIVPVYVND TSLLGGKSDIH ISPQQAN ISFYGLITQYPMCFSY & 143 \\
\hline $809 \mathrm{~T}$ & 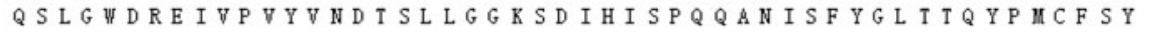 & 150 \\
\hline 83RS28 & QSLGWDREIVPVYVNDISLLGGKSDIHISPQQANISFYGLT TQYPMCFSY & 150 \\
\hline 84RS28 & QSLGWDREIVPVYVNDTSLLGGKSDIHISPQQAN ISFYGLTTQYPMCFSY & 143 \\
\hline $92 \mathrm{k} 3$ & QSLGWDREIVPVYVNDISLLGGKSDIHISPQQAN ISFYGLITQYP MCFSY & 150 \\
\hline JSRV21 & QS QHPHCI QVSADISYPRVTISGIDEKTGKKSYGNGSGPLDIPFCDKHLS & 193 \\
\hline JS7 & QSQHPHCIQVSADISYPRVT ISGIDEKTGKKSYGNGSGPLDIPFCDKHLS & 200 \\
\hline China-SD SPA & QSQHPHCIQVSADISYPRVIISGIDEKTGKKSYGNGSGPLDIPFCDKHLS & 193 \\
\hline $\mathrm{NM}$ & QS QHPHCIQVSADISYPRVTISGIDEKTGKKSYGNGSGPLDIPFCDKHLS & 193 \\
\hline JSRV-SA & QSQHPHCIQVSADISYPRVTISGIDEKTGKKSYGNGTGPLDIPFCDKHLS & 193 \\
\hline $809 \mathrm{~T}$ & QS QHPHCIQVSADISYPRVTISGIDEKT GKKSYGNGSGPLDIPFCDKHLS & 200 \\
\hline 83RS28 & QS QHPHCIQVSADISYPRVT ISGIDEKTGKKSYGNGSGPLDIPFCDKHLS & 200 \\
\hline 84RS28 & QSQHPHCIQVSADISYPRVT ISGIDEKTGKKSYGNGSGPLDIPFCDKHLS & 193 \\
\hline $92 \mathrm{k} 3$ & QS Q н P H C Q Q S D I SYPRVT ISGIDEKTGKKSYGNGTGPLDIPFCDKHLS & 200 \\
\hline JSRV21 & I G I G IDTP & 243 \\
\hline JS7 & I G I G I D T P T L CRARVASVYNINNANATFLWD & 250 \\
\hline China-SD SPA & I GIGIDTP TLCRARVAS VYNINNANATFL WD APGGTPDFPEYRGQHPP & 243 \\
\hline NM & 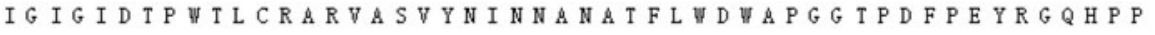 & 243 \\
\hline JSRV-SA & 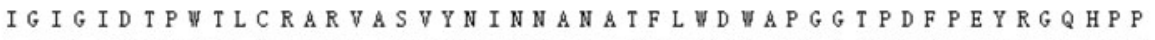 & 243 \\
\hline $809 \mathrm{~T}$ & I G I G ID T P T L CRARVASVYNINNANATFL D & 250 \\
\hline 83RS28 & 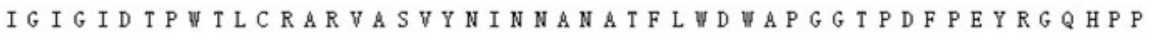 & 250 \\
\hline 84RS28 & I G I G I T P W L CRARVASVYNINNANATFL D A PGG TPDFAEYRGQHPP & 243 \\
\hline $92 \mathrm{k} 3$ & I G I I D T P T L CRARVASVYNINNANATFLWD A PGG TPDFPEYRGQHPP & 250 \\
\hline JSRV21 & IFSVN TAPIYQTEL KLLAAFGHGNSLYLQPNISGSKYGDVGVTGFLYPR & 293 \\
\hline JS7 & 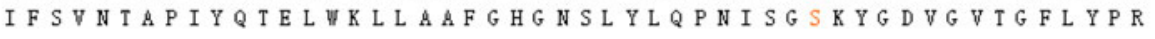 & 300 \\
\hline China-SD SPA & 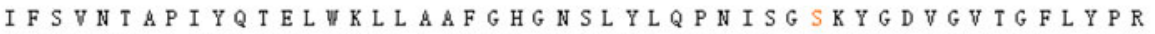 & 293 \\
\hline NM & IFSVN TAPIY Q TEL & 293 \\
\hline JSRV-SA & IFS VN TAPIYQTEL KLLA AFGHGNSLYLQPNISG TKYGDVGVTGFLYPR & 293 \\
\hline $809 \mathrm{~T}$ & 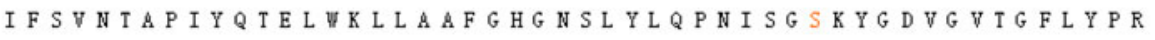 & 300 \\
\hline 83RS28 & IF $S \nabla N$ T \&P IYQTEL $\#$ KLL\&\&FGHGNSLYLQPNISGSKYGDVGVTGFLYPR & 300 \\
\hline 84RS28 & IFS VN TAPIYQAEL KLFA AFGHGNSLYLQPNISGSKYRDVGVTGFLYPR & 293 \\
\hline $92 \mathrm{k} 3$ & 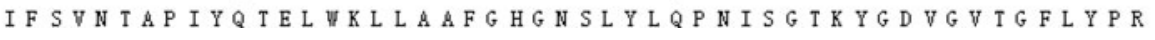 & 300 \\
\hline
\end{tabular}

Fig. 5 The boundary between the surface $(S U)$ and transmembrane $(T M)$ regions is indicated. The YXXM motif was highlighted by blue. VR3 is underlined; note the polymorphism between all sequences in VR3 (Color figure online) 
JS?

China-SD SPA

NM

JSRV-SA

$809 \mathrm{~T}$

83RS28

84RS28

$92 \mathrm{k} 3$

JSRV21
JS7

China-SD SPA

NM

JSRV-SA

$809 \mathrm{~T}$

83RS28

$84 \mathrm{RS} 28$

$92 \mathrm{k} 3$

JSRV21

JS?

China-SD SPA

NM

JSRV-SA

$809 \mathrm{~T}$

83RS28

84RS28

$92 \mathrm{k} 3$

JSRV21

JS7

China-SD SPA

NM

JSRV-SA

$809 \mathrm{~T}$

83RS28

84RS28

$92 \mathrm{k} 3$

JSRV 21

JS?

China-SD SPA

NM

JSRV-SA

$809 \mathrm{~T}$

83RS28

84RS28

$92 \mathrm{k} 3$

JSRV21

JS?

China-SD SPA

NM

JSRV-SA

$809 \mathrm{~T}$

83RS28

84RS28

$92 \mathrm{k} 3$
JSR21 ACVPYPFMLIQGHMEITLSLNIYHLNCSNCILTNCIRGVAKGEQVI I TKQ

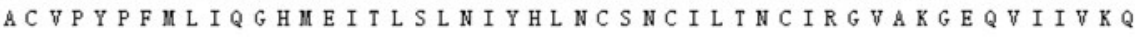
A C VP Y P F M I Q G H EITLSLNIYHLNCSNCILTNCIRGVAKGEQVIIVKQ A C VPYPFMLIQGHMEITLSLN I Y L N C SNCILINCIRGVAKGEQVIIVKQ A C VPYPFMLIQGHMEITLSLN I YHLNCSNCILINCIRGVAKGEQVIIVKQ

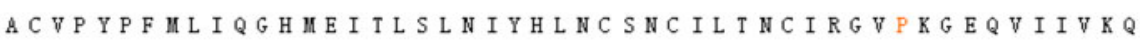
ACVPYPFMLIQGHMEITLSLNIYHLNCSNCILTNCIRGVAKGEQVIIVKQ A C VPYPFMLIQGHMEITLSLNIYHLNCSNCILINCIRGVAKGEQVIIVKQ A C VPYPFMLIQGHMEITLSLNIYHLNCSNCILTNCIRGVAKGEQVIIVKQ

\section{$\mathrm{SU} \longleftrightarrow \mathrm{TM}$}

PAFVML P VEIAEA W DETALELLQRINTALSRPKRGLSLIILGIVSLITL PAFVMLPVEIAEAWY DET ALELLQRINTALSRPKRGLSLIILGIVSLITL P A F V ML P E IAEAWY DETALELLQRIN TALSRPKRGLSLIILGIVSLITL PAFVMLPVEIAEAWYDETALELLQRINTALSRPKRGLSLIILGIVSLITL PAFVMLPVEIAEAWYDETALELLQRINIALSRPKRGLSLIILGIVSLIIL PAFVMLPVEITEAWYDETALELLQRIN TALSRPKRGLSLIILGIVSLITL PAFVMLPVEIAEAWYDETALELLQRINTALSRPRRGLSLIILGIVSLITL PAF M L P E I A A Y PAFVMLPVEIAEAWYDETALELLQRINTALSRPKRGLSLIILGIVSLITL

I A T A T A S V LAQSIQAAHTVDSLSYNVTKVMGTQEDIDKKIEDRLSALY IATAVTASVSLAQSIQAAHTVDSLSYNVTKVMGTQEDIDKKIEDRLSALY I A T A T A C V L A Q I Q A H T D L S Y N VTK M T TEDIDKKIEDRLSALY I A T A V T A S S L Q S Q A A H T D L S Y N V T KMG TQEDIDKKIEDRLSALY IATAVTACVSLAQSIQAAHTVDSLSTNVTKVMGTQEDIDKK IEDRLSALY I A T A T T S V LAQSIQAAHTVDSLSYNVTKVMGTQEDIDKKIEDRLSALY

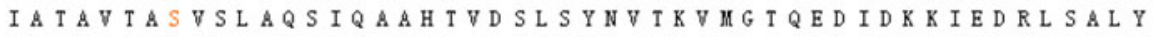
IA TAVTASVSLAQSIQAAHTVDSLSYNVTKVMGTQEDIDKKIEDRLSALY IA T A T T C V L A Q S Q A A T TDSLSYNVTKVMGTQEDIDKKIEDRLSALY

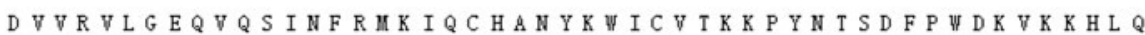
DVVR VLGEQVQSINFRMKIQCHAN YKWICVIKKPYNTSDFPWDKVKKHLQ DVVRVLGEQVQSINFRMKIQCHAN YKWICVIKKPYNTSDFPWDKVKKHLQ DVVRVLGEQVQSINFRMKIQCHANYKWICVTKKPYNTSDFPWDK VKKHLQ DVVRVLGEQVQSINFRMKIQCHANYKWICVIKKPYNTSDFPWDKVKKL Q DVVRVLGEQVQSINFRMKIQCHANYKWICVTKKPYNTSDFPW D V VRVLGEQVQSINFRMKIQCHANYKWICVIKKPYNTSDFP DKVKKHLQ DVVRVLGEQVQSINFRMKIQCHAN YKWICVTKRPYNTSDFPWDKVKKHLQ D V VRVLGEQVQSINFRMKIQCHAN YKWICVTKRPYNTSDFPW DKKKHLQ

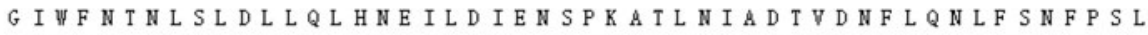

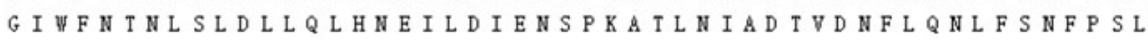

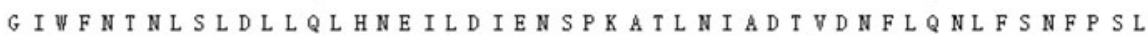

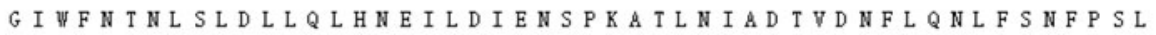

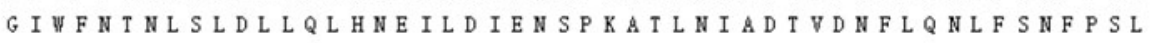

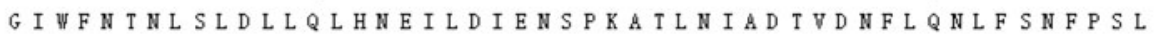
GIWFN TNLSLDLLQLHNEILDIENSPKA T LNIADT DNFLQNLFSNFPSL G IWFN T N L S D L L Q L H E I L D I ENSPKATLNIADTVDNFLQNLF SNF P S L GIWFN TNLSLDLLQLHNEILDIENSPKA TLNIADTVDNFLQNLFSNFP L VR3

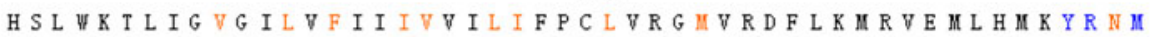
H SL WK TLIGVGILVFIIIVVILIFPCLVRGMVRDFLKMR VEMLHM Y Y R M HSL WK TLIGLGIFVIIIAIVIFVFPCV VRGLVRDFLKMRVEMLHMKYR T H SLWKTLLGLGILVFIIIVVILIFPCLVRG W R D F L KMR VEMLHM K R M M HSL W T L I GLG IFVIIIAIVIFVFPCVVRGLVRDFLKMRVEMLHMKYRTM HSLWK TLIGVGILVFIIIVVIPIFPCLVRG U RDFLKMRVEMLHMKYRNM HSLWK TLIG VGILVFIIIVVILIFPCLVRGMVRDFLKMRVEMLHMR YR M HSL WK TLIG VGILVFIIIVVILIFPCLVRGMVRDFLKMRVEMLHMKYRNM HSLWKTLIGLGIFVIIIAIVIFVFPCVVRGLVRDFLKMRVEMLHMKYR TM
343

350

343

343

343

350

350

343

350

393

400

393

393

393

400

400

393

400

443

450

443

443

443

450

450

443

450

493

500

493

493

493

500

500

493

500

543

550

543

543

543

550

550

543

550

593

600

593

593

593

600

600

593

600

Fig. 5 continued 


\begin{tabular}{|c|c|}
\hline JSRV21 & $L Q H Q H L M E L K N K E R G D A G D D P$ \\
\hline JS7 & $L Q H Q H L M E L K N K E R G D A G D D P$ \\
\hline China-SD SPA & L QHRHLMLLKNKERGA A G D P \\
\hline NM & 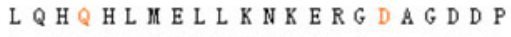 \\
\hline JSRV-SA & L Q H R H M E L L KNKE R A A G D D P \\
\hline $809 \mathrm{~T}$ & 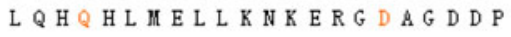 \\
\hline 83RS28 & L Q $H Q H L M E L K N K E R G D A D D P$ \\
\hline $84 \mathrm{RS} 28$ & L Q H Q H L M E L K N K ERGDRG T T R \\
\hline $92 \mathrm{k} 3$ & L Q H R H MEL L KNKER A A D D P \\
\hline
\end{tabular}

Fig. 5 continued all over the world. This article provided phylogenetic information about a JSRV strain in China, which will be of use for prospective studies of OPA in China.

Acknowledgments This study was supported by the National Natural Science Foundation of China (Grant No. 31201914) and the earmarked fund for China Agriculture Research System (CARS-39). The authors wish to thank the journal editors and anonymous reviewers for their editing to improve the manuscript.

Open Access This article is distributed under the terms of the Creative Commons Attribution License which permits any use, distribution, and reproduction in any medium, provided the original author(s) and the source are credited.

\section{References}

1. J.C. DeMartini, J.V. Bishop, T.E. Allen, F.A. Jassim, J.M. Sharp, M. De Las Heras et al., Jaagsiekte sheep retrovirus proviral clone JSRV(JS7), derived from the JS7 lung tumor cell line, induces ovine pulmonary carcinoma and is integrated into the surfactant protein a gene. J. Virol. 75(9), 4239-4246 (2001)

2. M. Palmarini, J.M. Sharp, M. De Las Heras, H. Fan, Jaagsiekte sheep retrovirus is necessary and sufficient to induce a contagious lung cancer in sheep. J. Virol. 73(8), 6964-6972 (1999)

3. H. Fan, M. Palmarini, J.C. DeMartini, Transformation and oncogenesis by jaagsiekte sheep retrovirus. Jaagsiekte Sheep Retrovir. Lung Cancer. 275, 139-177 (2003)

4. D.J. Griffiths, H.M. Martineau, C. Cousens, Pathology and pathogenesis of ovine pulmonary adenocarcinoma. J. Comp. Pathol. 142(4), 260-283 (2010)

5. M. De Las Heras, L. Gonzalez, J.M. Sharp, Pathology of ovine pulmonary adenocarcinoma. Jaagsiekte Sheep Retrovir. Lung Cancer 275, 25-54 (2003)

6. C. Cousens, L. Thonur, S. Imlach, J. Crawford, J. Sales, D.J. Griffiths, Jaagsiekte sheep retrovirus is present at high concentration in lung fluid produced by ovine pulmonary adenocarcinoma-affected sheep and can survive for several weeks at ambient temperatures. Res. Vet. Sci. 87(1), 154-156 (2009)

7. M. Palmarini, H. Fan, Retrovirus-induced ovine pulmonary adenocarcinoma, an animal model for lung cancer. J Natl Cancer I. 93(21), 1603-1614 (2001)

8. A. Hofacre, H. Fan, Jaagsiekte sheep retrovirus biology and oncogenesis. Viruses-Basel. 2(12), 2618-2648 (2010)

9. L. Xi, H. Xianpu, Z. Zhenhua, Y. Wangsheng, G. Yufang, B. Guopu, Pathological studies on intranasal adenoma and adenocarcinoma goats. Acta Vet Zootech Sin. 05, 456-461 (1995)
10. G. Yi, W. Kaiyu, Y. Qigui, Y. Zhongqiong, Y. Yingdong, C. Defang et al., Descriptive study of enzootic nasal adenocarcinoma in goats in southwestern China. Transbound. Emerg. Dis. 57(3), 197-200 (2010). Epub 2010/02/26

11. M. Palmarini, H. Fan, Molecular biology of jaagsiekte sheep retrovirus. Jaagsiekte Sheep Retrovir. Lung Cancer 275, 81-115 (2003)

12. F. Arnaud, M. Caporale, M. Varela, R. Biek, B. Chessa, A. Alberti et al., A paradigm for virus-host coevolution: sequential counter-adaptations between endogenous and exogenous retroviruses. PLoS pathog. 3(11), e170 (2007). Epub 2007/11/14

13. Y. Wang, S-y Liu, J-y Li, M. Han, Z-1 Wang, Cloning and sequence analysis of genome from the Inner Mongolia strain of the endogenous betaretroviruses (enJSRV). Virol. Sin. 23(1), 15-24 (2008)

14. M. Palmarini, C. Hallwirth, D. York, C. Murgia, T. de Oliveira, T. Spencer et al., Molecular cloning and functional analysis of three type D endogenous retroviruses of sheep reveal a different cell tropism from that of the highly related exogenous jaagsiekte sheep retrovirus. J. Virol. 74(17), 8065-8076 (2000). Epub 2000/08/10

15. M. Palmarini, N. Maeda, C. Murgia, C. De-Fraja, A. Hofacre, H. Fan, A phosphatidylinositol 3-kinase docking site in the cytoplasmic tail of the Jaagsiekte sheep retrovirus transmembrane protein is essential for envelope-induced transformation of NIH 3T3 cells. J. Virol. 75(22), 11002-11009 (2001). Epub 2001/10/17

16. F. Arnaud, P.R. Murcia, M. Palmarini, Mechanisms of late restriction induced by an endogenous retrovirus. J. Virol. 81(20), 11441-11451 (2007). Epub 2007/08/19

17. K. Voigt, M. Brugmann, K. Huber, P. Dewar, C. Cousens, M. Hall et al., PCR examination of bronchoalveolar lavage samples is a useful tool in pre-clinical diagnosis of ovine pulmonary adenocarcinoma (Jaagsiekte). Res. Vet. Sci. 83(3), 419-427 (2007)

18. A. Ortin, E. Minguijon, P. Dewar, M. Garcia, L.M. Ferrer, M. Palmarini et al., Lack of a specific immune response against a recombinant capsid protein of Jaagsiekte sheep retrovirus in sheep and goats naturally affected by enzootic nasal tumour or sheep pulmonary adenomatosis. Vet. Immunol. Immunop. 61(2-4), 229-237 (1998)

19. C. Summers, W. Neill, P. Dewar, L. Gonzalez, R. van der Molen, M. Norval et al., Systemic immune responses following infection with Jaagsiekte sheep retrovirus and in the terminal stages of ovine pulmonary adenocarcinoma. J. Gen. Virol. 83, 1753-1757 (2002)

20. T. Allen, Hematoxylin and eosin, in Laboratory methods in histotechnology, ed. by E.B. Prophet (Armed Forces Institute of Pathology, Washington, DC, 1992), pp. 53-58

21. R.G. Moore, C.O. Granai, W. Gajewski, M. Gordinier, M.M. Steinhoff, Pathologic evaluation of inguinal sentinel lymph nodes in vulvar cancer patients: a comparison of immunohistochemical staining versus ultrastaging with hematoxylin and eosin staining. Gynecol. Oncol. 91(2), 378-382 (2003). Epub 2003/11/06 
22. O. Rotimi, Vascular invasion is under recognized in colorectal cancer using conventional hematoxylin and eosin staining. Dis. Colon. Rectum 51(9), 1436-1437 (2008). author reply 8-9. Epub 2008/07/03

23. C. Wan, M. Dupeux, Preparation of cross-section specimens for transmission electron microscopy from alpha- $\mathrm{Al}_{2} \mathrm{O}_{3} / \mathrm{Ni}$ solid state bonded bicrystal interfaces. Microsc. Res. Tech. 23(3), 248-251 (1992)

24. Y.Z. Huang, S. Lozano-Perez, R.M. Langford, J.M. Titchmarsh, M.L. Jenkins, Preparation of transmission electron microscopy cross-section specimens of crack tips using focused ion beam milling. J. Microsc. 207(Pt 2), 129-136 (2002). Epub 2002/08/16

25. J.P. Clewley, Macintosh sequence analysis software. DNAStar's lasergene. Mol. Biotechnol. 3(3), 221-224 (1995)

26. T.G. Burland, DNASTAR's lasergene sequence analysis software. Methods. Mol. Biol. 132, 71-91 (2000). Epub 1999/11/05

27. J.D. Thompson, T.J. Gibson, F. Plewniak, F. Jeanmougin, D.G. Higgins, The CLUSTAL_X windows interface: flexible strategies for multiple sequence alignment aided by quality analysis tools. Nucleic Acids Res. 25(24), 4876-4882 (1997)

28. N. Saitou, M. Nei, The neighbor-joining method: a new method for reconstructing phylogenetic trees. Mol. Biol. Evol. 4(4), 406-425 (1987). Epub 1987/07/01

29. W. Zhang, Z. Sun, Random local neighbor joining: a new method for reconstructing phylogenetic trees. Mol. Phylogenet. Evol. 47(1), 117-128 (2008). Epub 2008/03/18

30. K. Tamura, J. Dudley, M. Nei, S. Kumar, MEGA4: molecular evolutionary genetics analysis (MEGA) software version 4.0. Mol. Biol. Evol. 24(8), 1596-1599 (2007). Epub 2007/05/10

31. M. Moos, I. Lemke, Risk assessment and risk management of veterinary biologicals with special regard to emerging diseases of horses, cattle and sheep in europe. Ecohealth 7, S165 (2011)

32. C. Leroux, N. Girard, V. Cottin, T. Greenland, J.F. Mornex, F. Archer, Jaagsiekte sheep retrovirus (JSRV): from virus to lung cancer in sheep. Vet. Res. 38(2), 211-228 (2007)

33. R. Tustin, Ovine jaagsiekte. J. S. Afr. Vet. Med. Assoc. 40, 3-23 (1969)

34. L. Shuying, M. Xueen, Resarch progresses on ovine pulmonary adenocarcinoma. Prog. Vet. Med. 24(1), 19-22 (2003)

35. D. Salvatori, L. Gonzalez, P. Dewar, C. Cousens, M. de Las Heras, R.G. Dalziel et al., Successful induction of ovine pulmonary adenocarcinoma in lambs of different ages and detection of viraemia during the preclinical period. J. Gen. Virol. 85(Pt 11), 3319-3324 (2004). Epub 2004/10/16

36. M. Caporale, P. Centorame, A. Giovannini, F. Sacchini, M. Di Ventura, M. de Las Heras et al., Infection of lung epithelial cells and induction of pulmonary adenocarcinoma is not the most common outcome of naturally occurring JSRV infection during the commercial lifespan of sheep. Virology 338(1), 144-153 (2005)
37. E. Grego, D. De Meneghi, V. Alvarez, A.A. Benito, E. Minguijon, A. Ortin et al., Colostrum and milk can transmit jaagsiekte retrovirus to lambs. Vet. Microbiol. 130(3-4), 247-257 (2008)

38. T.E. Besser, E.F. Cassirer, K.A. Potter, J. VanderSchalie, A. Fischer, D.P. Knowles et al., Association of mycoplasma ovipneumoniae infection with population-limiting respiratory disease in free-ranging rocky mountain bighorn sheep (Ovis canadensis canadensis). J. Clin. Microbiol. 46(2), 423-430 (2008)

39. B. Dungu, J. Vorster, G.F. Bath, D.W. Verwoerd, The effect of a natural maedi-visna virus infection on the productivity of South African sheep. Onderstepoort J. Vet. 67(2), 87-96 (2000)

40. F.A. Jassim, Identification and characterization of transformed cells in jaagsiekte, a contagious lung tumour of sheep (University of Edinburgh, New York, 1988)

41. M. Palmarini, J.M. Sharp, C. Lee, H. Fan, In vitro infection of ovine cell lines by Jaagsiekte sheep retrovirus. J. Virol. 73(12), 10070-10078 (1999). Epub 1999/11/13

42. A. Ortín, E. Minguijón, P. Dewar, M. García, L.M. Ferrer, M. Palmarini et al., Lack of a specific immune response against a recombinant capsid protein of Jaagsiekte sheep retrovirus in sheep and goats naturally affected by enzootic nasal tumour or sheep pulmonary adenomatosis. Vet. Immunol. Immunop. 61(2), 229-237 (1998)

43. S. Hull, H. Fan, Mutational analysis of the cytoplasmic tail of jaagsiekte sheep retrovirus envelope protein. J. Virol. 80(16), 8069-8080 (2006). Epub 2006/07/29

44. A. Hofacre, H. Fan, Multiple domains of the Jaagsiekte sheep retrovirus envelope protein are required for transformation of rodent fibroblasts. J. Virol. 78(19), 10479-10489 (2004). Epub 2004/09/16

45. Y.H.J. Chow, A. Alberti, M. Mura, C. Pretto, P. Murcia, L.M. Albritton et al., Transformation of rodent fibroblasts by the Jaagsiekte sheep retrovirus envelope is receptor independent and does not require the surface domain. J. Virol. 77(11), 6341-6350 (2003)

46. Z. Songyang, S.E. Shoelson, M. Chaudhuri, G. Gish, T. Pawson, W.G. Haser et al., SH2 domains recognize specific phosphopeptide sequences. Cell 72(5), 767-778 (1993). Epub 1993/03/12

47. S.L. Liu, F.M. Duh, M.I. Lerman, A.D. Miller, Role of virus receptor Hyal2 in oncogenic transformation of rodent fibroblasts by sheep betaretrovirus env proteins. J. Virol. 77(5), 2850-2858 (2003). Epub 2003/02/14

48. S.L. Liu, M.I. Lerman, A.D. Miller, Putative phosphatidylinositol 3-kinase (PI3K) binding motifs in ovine betaretrovirus Env proteins are not essential for rodent fibroblast transformation and PI3K/Akt activation. J. Virol. 77(14), 7924-7935 (2003). Epub 2003/06/28 